\title{
Usefulness of a Simple Protein-Energy Wasting Score for Predicting Hospitalization in Maintenance Hemodialysis Patients: A Prospective Cohort Study
}

\author{
Yoko Watanabe', Keiko Suzuki', Fumiko Kojima', Yoshiko Tanaka1, Kosaku Nitta2* \\ ${ }^{1}$ Shinjuku Ishikawa Clinic, Tokyo, Japan \\ ${ }^{2}$ Department of Nephrology, Tokyo Women's Medical University, Tokyo, Japan \\ Email: *nitta.kosaku@twmu.ac.jp
}

How to cite this paper: Watanabe, Y., Suzuki, K., Kojima, F., Tanaka, Y. and Nitta, K. (2021) Usefulness of a Simple Protein-Energy Wasting Score for Predicting Hospitalization in Maintenance Hemodialysis Patients: A Prospective Cohort Study. International Journal of Clinical Medicine, 12, 377-385.

https://doi.org/10.4236/ijcm.2021.129034

Received: August 23, 2021

Accepted: September 19, 2021

Published: September 22, 2021

Copyright $\odot 2021$ by author(s) and Scientific Research Publishing Inc. This work is licensed under the Creative Commons Attribution International License (CC BY 4.0).

http://creativecommons.org/licenses/by/4.0/

\section{(c) (i) Open Access}

\begin{abstract}
Background: Malnutrition is a strong predictor of prognosis in maintenance hemodialysis patients (MHD). We previously proposed a new and simple protein-energy wasting (PEW) score that appears to be useful for assessing the risk of mortality in patients on MHD. Objectives: In the present study, we evaluated the reliability of this PEW score as a predictor of hospitalization in Japanese patients on MHD. Methods: In this single-center, prospective cohort study conducted in Japan, PEW score was calculated for $180 \mathrm{MHD}$ patients. PEW score ranged from 0 (best: S1) to 4 (worst: S4) and was calculated based on nutritional indicators including serum albumin, body mass index, serum creatinine level, and protein intake. The outcome was the number of hospitalizations during the 2-year study period. Results: Thirty-six patients were hospitalized during the study period. Kaplan-Meier curves showed there were fewer hospitalizations in the group with a PEW score of $0 / 1$ than in the group with a score of $3 / 4$. Multivariate analysis revealed a hazard ratio for hospitalization of 3.109 for S3/4 versus S0, 2.777 for S3/4 versus S1, and 2.048 for S3/4 versus S2. Conclusion: The new and simple PEW score is a useful predictor of hospitalization in MHD patients and is also useful for identifying subgroups of MHD patients with a high risk of mortality.
\end{abstract}

\section{Keywords}

Protein-Energy Wasting, Hemodialysis, Hospitalization, Simple Score

\section{Introduction}

Patients on maintenance hemodialysis (MHD) have various nutritional disord- 
ers due to the stepwise loss of body proteins and metabolic disorders [1]. Because malnutrition is a strong predictor of mortality in MHD patients, assessing their nutritional status is essential in managing these patients [2]. In 2008, the International Society of Renal Nutrition and Metabolism (ISRNM) presented the concept of protein energy wasting (PEW) and proposed diagnostic criteria for PEW in MHD patients [3]. However, the proposed approach lacks versatility because it is difficult to assess the reduced muscle mass. To address this issue, a new and simplified scoring method using serum creatinine adjusted for body surface area (SCr/BSA) was proposed in 2014 [4].

Currently, there is no single gold standard marker for clinically evaluating nutritional status in MHD patients that are not affected by confounding factors and that can be measured by a method that is both simple and reproducible. Therefore, PEW has conventionally been evaluated based on the assessment of various factors. We previously proposed a new and simple PEW score that appears to be useful for assessing the risk of mortality in patients on MHD [5]. In the present study, we aimed to demonstrate the usefulness of this simple PEW score as a predictor of hospitalization in MHD patients.

\section{Methods}

\subsection{Patients and Protocol}

This was a single-center, prospective cohort study conducted over 24 months in Japan from June 1, 2017, to June 30, 2019, at Shinjuku Ishikawa Clinic. The study protocol complied with the Declaration of Helsinki and was approved by the Institutional Review Board of Ishikawa Clinic (No. 2-01-2017). All subjects gave informed consent to participate.

This study enrolled MHD patients who had been undergoing hemodialysis (HD) via an arteriovenous fistula (AVF) for at least 6 months at Shinjuku Ishikawa Clinic. Exclusion criteria were malignancy, active inflammation, liver cirrhosis, gastrointestinal bleeding, and other severe illness.

All patients received HD 3 times a week. Blood pressure was measured in the recumbent position in a resting state, and the mean blood pressure during the 1-month period prior to enrollment was used for the analysis. The dry weight was targeted so as to achieve a normotensive edema-free state. Diabetes mellitus was defined as fasting blood glucose of $\geq 126 \mathrm{mg} / \mathrm{dL}$, hemoglobin A1c (HbA1c) of $\geq 6.5$, or a history of medication with an oral hypoglycemic agent(s).

\subsection{Laboratory and Nutritional Parameters}

Blood sampling was performed before starting an HD session after overnight fasting. Blood urea nitrogen and serum creatinine, albumin, and C-reactive protein (CRP) were measured using an autoanalyzer (Hitachi Co., Tokyo, Japan). Body mass index (BMI) was calculated by dividing body weight in kilograms by height squared in meters. Urea kinetics was assessed by measuring the bloodbased dialysis parameter, $\mathrm{Kt} / \mathrm{V}[6]$, and the mean value of three measurements 
obtained during each of the 3 months before the start of the study was used in the analysis.

PEW score was determined by grading one selected item in each of four categories, as previously described [5]: 1) serum albumin, 2) BMI, 3) predialysis SCr/BSA, and 4) normalized protein catabolic rate (nPCR). The threshold values used were serum albumin $<3.8 \mathrm{~g} / \mathrm{dL}, \mathrm{BMI}<23 \mathrm{~kg} / \mathrm{m}^{2}, \mathrm{SCr} / \mathrm{BSA}<659 \mathrm{mmol} / \mathrm{L} / \mathrm{m}^{2}$, and $\mathrm{nPCR}<0.8 \mathrm{~g} / \mathrm{kg} /$ day. $\mathrm{nPCR}$ was used as an indirect indicator of protein intake and was calculated using a formula previously reported [7]. BSA was estimated using the $\mathrm{Du}$ Bois formula [8]. A threshold value of $659 \mathrm{mmol} / \mathrm{L} / \mathrm{m}^{2}$ for the SCr/BSA variable was selected based on the results of a receiver operating characteristics (ROC) curve analysis (Figure 1).

\subsection{Study Outcome}

Data for endpoints were obtained from hospital charts. The primary endpoint of the study was hospitalization. The following hospitalization data were collected: 1) date of hospitalization and discharge, 2) diagnosis, and 3) treatment. This study was a prospective study and the sample size was dependent on the number of HD patients eligible for enrollment in this study.

\subsection{Statistical Analysis}

Nonparametric values were expressed as median values and compared using the Kruskal-Wallis test. Categorical values were expressed as percentages and compared using Fisher's exact test. In univariate logistic regression, we determined variables with a $P$-value of $<0.10$, in addition to sex, presence of diabetes, CRP, and $\mathrm{Kt} / \mathrm{V}$. Hospitalization was analyzed based on the Kaplan-Meier curve. A log-rank test was used to compare the hospitalization rates of two groups. A multivariate Cox proportional hazard model with adjustment for multivariate factors was used to evaluate risk of hospitalization. Results are expressed as hazard ratios (HRs) with 95\% confidence intervals (CIs). Statistical significance was set at $P<0.05$. All statistical analyses were performed using SAS version 9.2 software program (SAS Institute Inc., Cary, NC) for Windows personal computers. There were no missing data or loss to follow-up in this study.

\section{Results}

A total of 180 patients on stable HD maintained with a bicarbonate dialysate were enrolled in the study. The baseline characteristics of the participants classified using the PEW score are shown in Table 1 . The study cohort consisted of 38 women and 142 men (mean age 61 years; mean dialysis vintage 10 years; mean BMI 21.8). The underlying kidney diseases were chronic glomerulonephritis (74 patients), diabetic kidney disease (62 patients), glomerulosclerosis (32 patients), and other (12 patients). None had any residual renal function (urine volume < $100 \mathrm{~mL}$ ). Mean dialysis dose was 1.49 for a single-pool Kt/V, and mean protein intake was $1.12 \mathrm{~g} / \mathrm{kg} /$ day. Of the 180 patients, 102 (56.7\%) had moderate or se- 
vere wasting. Data for all participants were available for analysis during the follow-up period. There were no missing data in this study.

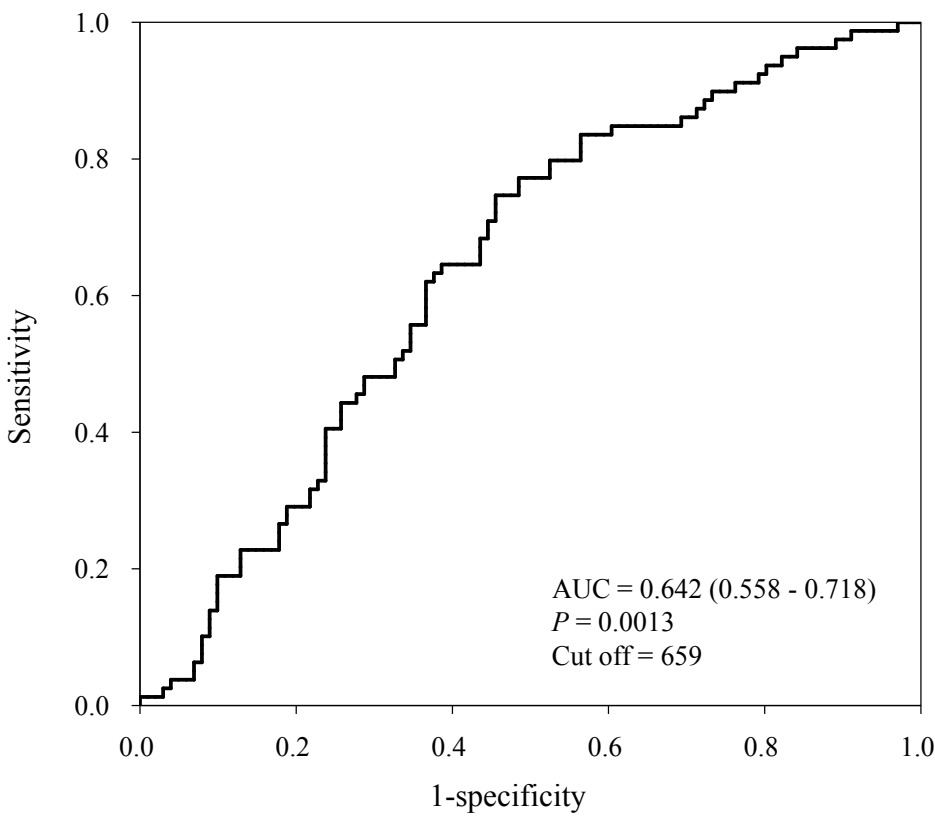

Figure 1. Receiver operating characteristic curve analysis to determine the optimal cut-off value of serum creatinine adjusted for body surface area for detecting hospitalization rate. Abbreviation: AUC, area under the curve.

Table 1. Baseline characteristics according to the protein-energy wasting score.

\begin{tabular}{cccccc}
\hline & & Score 0 & Score 1 & Score 2 & Score $3-4$ \\
\hline $\begin{array}{c}\text { Clinical and laboratory } \\
\text { parameters }\end{array}$ & All & $\begin{array}{c}\text { Normal nutritional } \\
\text { status }\end{array}$ & Slight wasting & Moderate wasting & Severe wasting \\
\hline Number of patients & 180 & 18 & 60 & 65 & 37 \\
Age (years) & $61(51-69)$ & $50(45-57)$ & $55(48-65)$ & $61(53-70)^{* *}$ & $73(66-80)^{*}$ \\
Male (\%) & $142(78.9)$ & $16(88.9)$ & $52(86.7)$ & $53(81.5)$ & $21(56.8)$ \\
Dialysis vintage (years) & $10(4-17)$ & $10(7-14)$ & $10(3-18)$ & $13(5-19)$ & $9(4-17)$ \\
BMI (kg/m $)$ & $21.8(19.9-24.7)$ & $26.4(24.1-27.2)$ & $21.9(20.1-24.9)^{*}$ & $21.6(20-24.5)^{*}$ & $20.4(18.8-21.7)^{*}$ \\
Kt/V & $1.49(1.36-1.61)$ & $1.36(1.24-1.56)$ & $1.46(1.36-1.58)$ & $1.51(1.34-1.61)$ & $1.60(1.40-1.78)^{* *}$ \\
nPCR (g protein/kg/day) & $1.12(0.97-1.27)$ & $1.05(0.97-1.23)$ & $1.15(0.99-1.35)$ & $1.13(0.96-1.29)$ & $1.07(0.88-1.20)$ \\
Hb (g/dL) & $11.2(10.8-11.7)$ & $11.4(10.9-12.0)$ & $11.3(10.9-11.7)$ & $11.1(10.8-11.6)$ & $11(10.2-11.8)$ \\
Serum albumin $(\mathrm{g} / \mathrm{dL})$ & $3.8(3.5-4.0)$ & $4.0(3.9-4.1)$ & $3.9(3.8-4.1)$ & $3.7(3.5-3.8)^{*}$ & $3.5(3.4-3.6)^{*}$ \\
Pre-albumin $(\mathrm{mg} / \mathrm{dL})$ & $35.1(29.4-40.9)$ & $41.2(37.9-51.2)$ & $38.6(34.2-42.3)$ & $33.9(29.5-39.1)^{*}$ & $27.8(23.15-32.6)^{*}$ \\
Creatinine/BSA $\left(\mu \mathrm{mol} / \mathrm{L} / \mathrm{m}^{2}\right)$ & $635.8(542.6-732.9)$ & $780.2(688.7-816.0)$ & $709.7(604.4-774.5)^{* *}$ & $604.6(528.2-688.4)^{*} 556.6(488.7-601.7)^{*}$ \\
C-reactive protein $(\mathrm{mg} / \mathrm{dL})$ & $0.10(0.05-0.27)$ & $0.13(0.05-0.21)$ & $0.07(0.05-0.20)$ & $0.08(0.05-0.27)$ & $0.21(0.05-0.67)$ \\
Total cholesterol $(\mathrm{mg} / \mathrm{dL})$ & $153(133-169)$ & $159(140-181)$ & $157(140-173)$ & $152(135-167)$ & $147(127-164)$ \\
HDL cholesterol $(\mathrm{mg} / \mathrm{dL})$ & $45(36-54)$ & $40(33-50)$ & $49(40-60)$ & $44(35-53)$ & $44(36-50)$ \\
Triglyceride $(\mathrm{mg} / \mathrm{dL})$ & $93(62-151)$ & $158(135-182)$ & $99(76-146)^{* *}$ & $86(57-141)^{* *}$ & $81(59-121)^{* *}$ \\
\hline
\end{tabular}

Abbreviations: BMI, body mass index; Kt/V, blood-based dialysis parameter; nPCR, normalized protein catabolic rate; HDL, high-density lipoprotein; BSA, body surface area. ${ }^{*} P<0.001$ versus score $0 ;{ }^{*} P<0.05$ versus score 0 . 
There were 168 hospitalizations during the 24-month follow-up period. The most common cause of hospitalization was pneumonia. Kaplan-Meier curve analysis revealed that the hospitalization rate was lower in the group with a PEW score of 0 (S0) than in the group with a high PEW score (S2-4; Figure 2). Table 2 shows predictors of hospitalization in the MHD patients. The HRs for hospitalization in the severe wasting group (S3/4) relative to the normal nutritional status group (S0), slight wasting group (S1) and moderate wasting group (S2) were $3.109,2.774$, and 2.048, respectively (Table 2 ).

Table 2. Predictive factors of hospitalization in patients on maintenance hemodialysis as determined by Cox proportional hazard models.

\begin{tabular}{cccc}
\hline Patient characteristics and PEW score & HR & $95 \%$ CI & $P$-value \\
\hline Sex (male/female) & 3.317 & $1.522-7.629$ & 0.0022 \\
Diabetes & 2.252 & $1.354-3.777$ & 0.0018 \\
Myocardial infarction & 1.309 & $0.492-2.909$ & 0.5586 \\
Peripheral vascular disease & 1.709 & $0.402-4.952$ & 0.4185 \\
Stroke & 1.636 & $0.907-2.821$ & 0.0992 \\
Dialysis vintage & 0.998 & $0.967-1.028$ & 0.8760 \\
C-reactive protein & 1.138 & $0.914-1.317$ & 0.1391 \\
Kt/V & 4.795 & $1.342-16.940$ & 0.0154 \\
Score 3 - 4 versus 0 & 3.109 & $1.220-9.551$ & 0.0160 \\
Score 3 - 4 versus 1 & 2.774 & $1.423-5.439$ & 0.0029 \\
Score 3 - 4 versus 2 & 2.048 & $1.141-3.624$ & 0.0169 \\
\hline
\end{tabular}

Abbreviations: PEW score, protein-energy wasting score; HR, hazards ratio; CI, confidence interval; Kt/V, blood-based dialysis parameter.

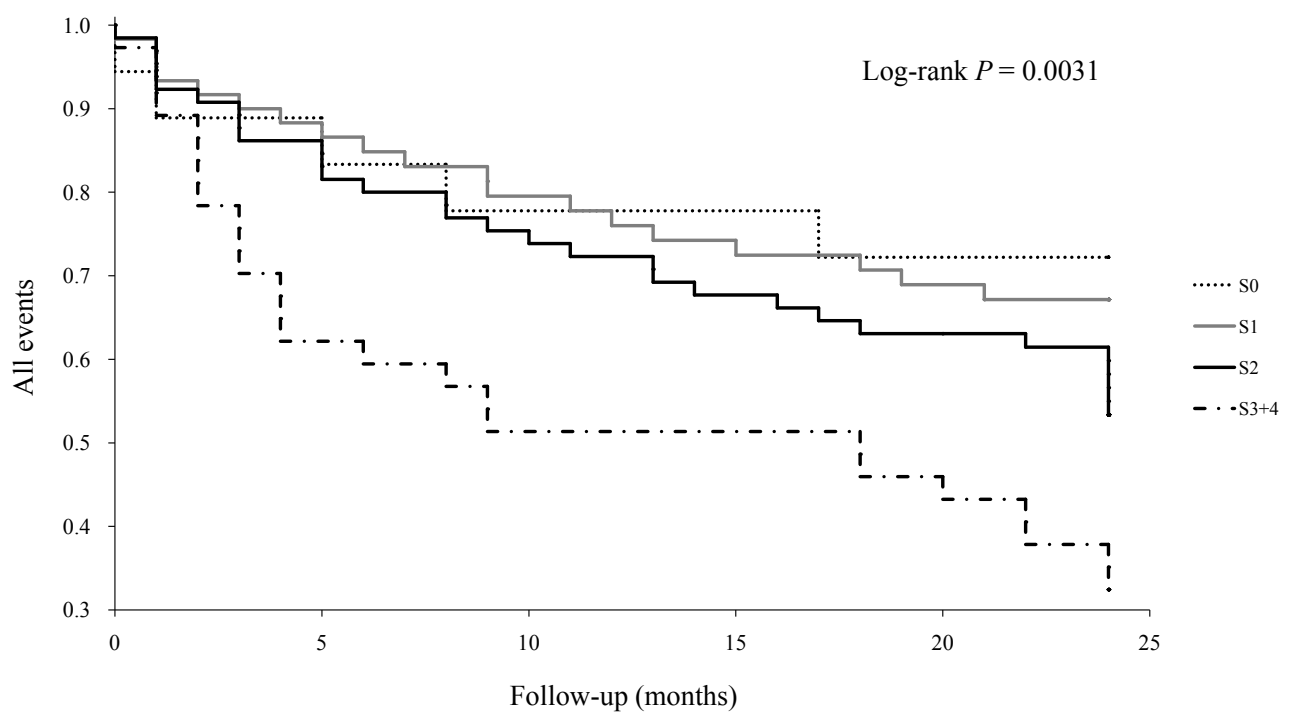

Figure 2. Kaplan-Meyer curve for hospitalization according to the protein-energy wasting score. S0 had a normal nutritional status, S1 had slight wasting, S2 had moderate wasting, and S3-4 had severe wasting. 


\section{Discussion}

We evaluated the usefulness of a simple PEW score calculated from readily available clinical parameters and demonstrated its utility as a predictor of the risk of hospitalization in MHD patients. Although impaired nutritional status is frequently reported in MHD patients, there is no single nutritional parameter that can predict PEW. We hope to improve outcomes in MHD patients by using simple nutritional markers to evaluate their nutritional status.

Hypoalbuminemia is a strong predictor of mortality in MHD patients. Kalantar-Zadeh et al. showed that a serum albumin level of $<3.8 \mathrm{~g} / \mathrm{dL}$ in MHD patients was correlated with increased mortality from cardiovascular disease, independent of demographic, clinical and hematological data [9]. Also, Malfra et al. demonstrated that serum albumin level of $<3.7 \mathrm{~g} / \mathrm{dL}$ was a strong predictor of mortality in HD patients [10]. A 10-year cohort study reported increased risk of mortality in HD patients with a serum albumin level of $\leq 3.8 \mathrm{~g} / \mathrm{dl}$ [11]. However, Friedman and Fadem [12] recently showed that the serum albumin level should be used cautiously as a nutritional marker in dialysis patients, because hypoalbuminemia could arise from both malnutrition and inflammation.

A lower prevalence of inflammation has been reported in HD patients in Asian countries, including Japan and Korea, and this lower prevalence may be associated with genetic factors and cultural habits, such as dietary factors [13] [14]. The prevalence of obesity in MHD patients appears to be paradoxically associated with a higher survival rate [15] [16]. Mortality in Asian-American HD patients is reported to be lower than that in Caucasian HD patients. In fact, Asian-Americans have a significantly lower BMI [17]. The ISRNM has proposed a BMI of $\leq 23$ as a diagnostic criterion for PEW in patients with chronic kidney disease (CKD) patients [3], but has not recommended its use in South Asian CKD patients [5].

nPCR reflects dietary protein intake [18], and it was reported to be an independent predictor of mortality in MHD patients [19]. A study by Chandna et al. showed a substantial decrease in nPCR among CKD patients at 3 months before the start of dialysis [20]. The Kidney/Dialysis Outcomes Quality Initiative (K/DOQI) clinical practice guidelines recommend a daily protein intake of 1.2 $1.3 \mathrm{~g} / \mathrm{kg} /$ day for MHD patients [21]. Lukowsky et al. reported that patients with nPCR decreased by $\geq 0.2$ within 3 months before the start of dialysis have an increased risk of death [22].

No single parameter allows for comprehensive and conclusive assessment of nutritional status in HD patients. Therefore, collective evaluation of multiple nutritional markers is recommended by the K/DOQI guidelines [21]. A recent expert panel has suggested the use of markers from four different categories (blood biochemistry, BMI, muscle mass, and dietary intake) for the clinical diagnosis of PEW [3]. Three out of these four categories should be selected, including at least one biochemical factor, to satisfy this diagnosis. However, to our knowledge, these combinations have not yet been tested for the assessment of nutritional status in MHD patients. 
Moreau-Gaudry et al. [4] reported a simple PEW scoring method that includes one parameter from each of the following major groups proposed for nutritional intervention: 1) biological parameters, 2) body composition, 3) muscle mass, and 4) nutrient intake. It seems important to add hematological data (e.g., serum albumin and serum creatinine) and other clinical information (e.g., BMI). Muscle mass represents an important body feature strongly associated with survival, but because assessment of muscle mass is difficult, predialysis SCr/BSA was used for the assessment. SCr/BSA values differ between Western and Asian subjects, and may vary depending on creatinine uptake and metabolism. This is why serum creatinine is not used routinely. In fact, SCr/BSA shows a better fit to the Cox model than serum albumin. Eventually, we decided to include protein intake, estimated by the nPCR, as information on nutrient intake in the score. This value is also listed in the recent international recommendations and can be easily calculated using dialysis generator software. The inclusion of SCr/BSA in our model improved the prediction of hospitalization compared with the use of albumin alone.

Our study has several limitations. First, because some participants had comorbid conditions, the way in which the study population was enrolled may have introduced selection bias and may have failed to gather a representative sample of the general Japanese population. Second, it should be noted that the score itself was not better than the four parameters included in the model as separate variables [4]. However, this result was not unexpected, because the information given by the score depends on the variables included in the score. We believe that this score is of interest because it would encourage dialysis staff to pay more attention to all of these variables. The PEW score can be calculated within minutes at the bedside and requires no additional preparation or expense. The final patient classification we obtained corresponds well to a publication on the prevalence of nutritional disorders in MHD patients, which reports that moderate and severe nutritional disorders are found in $37 \%$ and $19 \%$ of HD patients, respectively [23].

\section{Conclusion}

This simple-to-calculate nutritional score is useful for the identification of PEW in MHD patients in routine clinical practice. Further studies are needed to establish the PEW score in Asian populations because of differences in body composition and clinical practice.

\section{Acknowledgements}

The authors are grateful to the medical staff of Shinjuku Ishikawa Clinic who provided high-quality data.

\section{Conflicts of Interest}

The authors declare no conflicts of interest regarding the publication of this paper. 


\section{References}

[1] Kalantar-Zadeh, K., Ikizler, T.A., Block, G., Avram, M.M. and Kopple, J.D. (2003) Malnutrition-Inflammation Complex in Dialysis Patients: Causes and Consequences. American Journal of Kidney Diseases, 42, 864-881. https://doi.org/10.1016/j.ajkd.2003.07.016

[2] Lopes, A.A., Bragg-Gresham, J.L., Elder, S.J., Ginsberg, N., Goodkin, D.A., Pifer, T., Lameire, N., Marshall, M.R., Asano, Y., Akizawa, T., Pisoni, R.L., Young, E.W. and Port, F.K. (2010) Independent and Joint Associations of Nutritional Status Indicators with Mortality Risk among Chronic Hemodialysis Patients in the Dialysis Outcomes and Practice Patterns Study (DOPPS). Journal of Renal Nutrition, 20, 224-234. https://doi.org/10.1053/j.jrn.2009.10.002

[3] Fouque, D., Kalantar-Zadeh, K., Kopple, J., Cano, N., Chauveau, P., Cuppari, L., Franch, H., Guamieri, G., Ikizler, T.A., Kaysen, G., Lindholm, B., Massy, Z., Mitch, W., Pineda, E., Stenvinkel, P., Trevino-Becerra, A. and Wanner, C. (2008) A Proposed Nomenclature and Diagnostic Criteria for Protein-Energy Was Ting in Acute and Chronic Kidney Disease. Kidney International, 73, 391-398. https://doi.org/10.1038/sj.ki.5002585

[4] Moreau-Gaudry, X., Jean, G., Genet, L., Lataillade, D., Legrand, E., Kuentz, F. and Fouque, D. (2014) A Simple Protein-Energy Wasting Score Predicts Survival in Maintenance Hemodialysis Patients. Journal of Renal Nutrition, 24, 395-400. https://doi.org/10.1053/j.jrn.2014.06.008

[5] Kobayashi, S., Suzuki, K., Ueda, M., Tanaka, Y. and Nitta, K. (2015) A Simple Protein-Energy Wasting Score for Survival Prediction of Maintenance Hemodialysis Patients. Renal Replacement Therapy, 1, Article No. 1.

https://doi.org/10.1186/s41100-015-0012-0

[6] Daugirdas, J.T. (1993) Second Generation Logarithmic Estimates of Single-Pool Variable Volume Kt/V: An Analysis of Error. Journal of American Society of Nephrology, 4, 1205-1213. https://doi.org/10.1681/ASN.V451205

[7] Shinzato, T., Nakai, S., Fujita, Y., Takai, I., Morita, H., Nakane, K. and Maeda, K. (1994) Determination of Kt/V and Protein Catabolic Rate Using Pre- and Postdialysis Blood Urea Nitrogen Concentrations. Nephron, 67, 280-290. https://doi.org/10.1159/000187980

[8] Du Bois, D. and Du Bois, E.F. (1989) A Formula to Estimate the Approximate Surface Area If Height and Weight Be Known. Nutrition, 5, 303-311.

[9] Kalantar-Zadeh, K., Kilpatrick, R.D., Kuwae, N., McAllister, C.J., Alcom, H.Jr., Kopple, J.D. and Greenland, S. (2005) Revising Mortality Predictability of Serum Albumin in the Dialysis Population: Time Dependency, Longitudinal Changes and Population-Attributable Fraction. Nephrology Dialysis Transplantation, 9, 1880-1888. https://doi.org/10.1093/ndt/gfh941

[10] Mafra, D., Farage, N.E., Azevedo, D.L., Viana, G.G., Mattos, J.P., Velarde, L.G.C. and Fouque, D. (2007) Impact of Serum Albumin and Body-Mass Index on Survival in Hemodialysis Patients. International Urology and Nephrology, 39, 619-624. https://doi.org/10.1007/s11255-007-9201-2

[11] Kato, A., Takita, T., Furuhashi, M., Maruyama, Y. and Hishida, A. (2010) Comparison of Serum Albumin, C-Reactive Protein and Carotid Atherosclerosis as Predictors of 10-Year Mortality in Hemodialysis Patients. Hemodialysis International, 14, 226-232. https://doi.org/10.1111/j.1542-4758.2009.00432.x

[12] Friedman, A.N. and Fadem, S.Z. (2010) Reassessment of Albumin as a Nutritional Marker in Kidney Disease. Journal of American Society of Nephrology, 21, 223-230. 
https://doi.org/10.1681/ASN.2009020213

[13] Oldroyd, J.C., Heald, A., Bansal, N., Vyas, A., Siddals, K., Gibson, M., Clayton, P. and Cruickshamk, J.K. (2009) Inflammatory Markers and Growth in South Asian and European Origin Infants in Britain: The Manchester Children's Growth and Vascular Health Study. Atherosclerosis, 207, 227-231. https://doi.org/10.1016/j.atherosclerosis.2009.03.045

[14] Coe, C.L., Love, G.D., Karasawa, M., Kawakami, N., Kitayama, S., Markus, H.R., Tracy, R.P. and Ryff, C.D. (2011) Population Differences in Proinflammatory Biology: Japanese Have Healthier Profiles than Americans. Brain Behavior and Immunology, 25, 494-502. https://doi.org/10.1016/j.bbi.2010.11.013

[15] Kalantar-Zadeh, K., Kopple, J.D., Kilpatrick, R.D., McAllister, C.J., Shinaberger, C.S., Gjertson, D.W. and Greenland, S. (2005) Associations of Morbid Obesity and Weight Change over Time with Cardiovascular Survival in Hemodialysis Patients. American Journal of Kidney Diseases, 46, 489-500.

https://doi.org/10.1053/j.ajkd.2005.05.020

[16] Kalantar-Zadeh, K., Kuwae, N., Wu, D.Y., Shantouf, R.S., Fouque, D., Anker, S.D., Block, F. and Kopple, J.D. (2006) Associations of Body Fat and Its Changes over Time with Quality of Life and Prospective Mortality in Hemodialysis Patients. American Journal of Clinical Nutrition, 83, 202-210.

https://doi.org/10.1093/ajcn/83.2.202

[17] Wong, J.S., Port, F.K., Hulbert-Shearon, T.E., Carroll, C.E., Wolfe, R.A., Agodoa, L.Y. and Daugirdas, J.T. (1999) Survival Advantage in Asian American End-Stage Renal Disease Patients. Kidney International, 55, 2515-2523. https://doi.org/10.1046/j.1523-1755.1999.00464.x

[18] Shinaberger, C.S., Kilpatrick, R.D., Regidor, D.L., McAllister, C.J., Greenland, S., Kopple, J.D. and Kalantar-Zadeh, K. (2006) Longitudinal Associations between Dietary Protein Intake and Survival in Hemodialysis Patients. American Journal of Kidney Diseases, 48, 37-49. https://doi.org/10.1053/j.ajkd.2006.03.049

[19] Mancini, A., Grandaliano, G., Magarelli, P. and Allegretti, A. (2003) Nutritional Status in Hemodialysis Patients and Bioimpedance Vector Analysis. Journal of Renal Nutrition, 13, 199-204. https://doi.org/10.1016/S1051-2276(03)00079-7

[20] Chandra, S.M., Kulinskaya, E. and Farrington, K. (2005) A Dramatic Reduction of Normalized Protein Catabolic Rate in the Course of Progressive Renal Insufficiency. Nephrology Dialysis Transplantation, 20, 2130-2138.

https://doi.org/10.1093/ndt/gfh940

[21] K/DOQI (200) National Kidney Foundation. Clinical Practice Guidelines for Nutrition in Chronic Renal Failure. American Journal of Kidney Diseases, 35, S1-S140.

[22] Lukowsky, L.P., Khefets, L., Arah, O.A., Nissenson, A.R. and Kalantar-Zadeh, K. (2014) Nutritional Predictors of Early Mortality in Incident Hemodialysis Patients. International Urology and Nephrology, 46, 129-140. https://doi.org/10.1007/s11255-013-0459-2

[23] Dukkipati, R. and Kopple, J.D. (2009) Causes and Prevention of Protein-Energy Wasting in Chronic Renal Failure. Seminars in Nephrology, 29, 39-49. https://doi.org/10.1016/j.semnephrol.2008.10.006 